\title{
Hyperglycemia exacerbates
} acetaminophen-induced acute liver injury by promoting liver-resident macrophage proinflammatory response via AMPK/PI3K/ AKT-mediated oxidative stress

\author{
Qi Wang $\mathbb{1}^{1}$, Song Wei ${ }^{1,2}$, Haoming Zhou', Gefenqiang Shen', Xiaojie Gan', Shun Zhou', Jiannan Qiu',
} Chenyu Shi ${ }^{1}$ and Ling Lư ${ }^{1,2}$

\begin{abstract}
Although diabetes mellitus/hyperglycemia is a risk factor for acute liver injury, the underlying mechanism remains largely unknown. Liver-resident macrophages (Kupffer cells, KCs) and oxidative stress play critical roles in the pathogenesis of toxin-induced liver injury. Here, we evaluated the role of oxidative stress in regulating KC polarization against acetaminophen (APAP)-mediated acute liver injury in a streptozotocin-induced hyperglycemic murine model. Compared to the controls, hyperglycemic mice exhibited a significant increase in liver injury and intrahepatic inflammation. KCs obtained from hyperglycemic mice secreted higher levels of the proinflammatory factors, such as TNF-a and IL-6, lower levels of the anti-inflammatory factor IL-10. Furthermore, enhanced oxidative stress was revealed by increased levels of reactive oxygen species (ROS) in KCs from hyperglycemic mice post APAP treatment. In addition, ROS inhibitor NAC resulted in a significant decrease of ROS production in hyperglycemic KCs from mice posttreated with APAP. We also analyzed the role of hyperglycemia in macrophage M1/M2 polarization. Interestingly, we found that hyperglycemia promoted M1 polarization, but inhibited M2 polarization of KCs obtained from APAP-exposed livers, as evidenced by increased MCP-1 and inducible NO synthase (iNOS) gene induction but decreased Arg-1 and CD206 gene induction accompanied by increased STAT1 activation and decreased STAT6 activation. NAC restored Arg-1, CD206 gene induction, and STAT6 activation. To explore the mechanism how hyperglycemia regulates KCs polarization against APAP-induced acute liver injury, we examined the AMPK/PI3K/AKT signaling pathway and found decreased AMPK activation and increased AKT activation in liver and KCs from hyperglycemic mice post APAP treatment. AMPK activation by its agonist AICAR or PI3K inhibition by its antagonist LY294002 inhibited ROS production in KCs from hyperglycemic mice post APAP treatment and significantly attenuated APAP-induced liver injury in the hyperglycemic mice, compared to the control mice. Our results demonstrated that hyperglycemia exacerbated APAP-induced acute liver injury by promoting liverresident macrophage proinflammatory response via AMPK/PI3K/AKT-mediated oxidative stress.
\end{abstract}

Correspondence: Ling Lu (Ivling@njmu.edu.cn)

${ }^{1}$ Liver Transplantation Center, The First Affiliated Hospital of Nanjing Medical University, Key Laboratory of Liver Transplantation, Chinese Academy of Medical Sciences, NHC Key Laboratory of Living Donor Liver Transplantation, Nanjing, China

${ }^{2}$ School of Medicine, Southeast University, Nanjing, China

These authors contributed equally: Qi Wang, Song Wei

Edited by A. Ruffini

\section{Introduction}

Diabetes mellitus is a metabolic disorder characterized by hyperglycemia and high morbidity worldwide ${ }^{1}$. Studies revealed that hyperglycemia aggravated acute liver injury $^{2,3}$. The detrimental effects of hyperglycemia include an increase in the oxidative stress response and an

\section{(c) The Author(s) 2019}

(c) (i) Open Access This article is licensed under a Creative Commons Attribution 4.0 International License, which permits use, sharing, adaptation, distribution and reproduction cc. in any medium or format, as long as you give appropriate credit to the original author(s) and the source, provide a link to the Creative Commons license, and indicate if changes were made. The images or other third party material in this article are included in the article's Creative Commons license, unless indicated otherwise in a credit line to the material. If material is not included in the article's Creative Commons license and your intended use is not permitted by statutory regulation or exceeds the permitted use, you will need to obtain permission directly from the copyright holder. To view a copy of this license, visit http://creativecommons.org/licenses/by/4.0/. 
enhanced inflammatory response ${ }^{1}$. Acetaminophen (APAP) is a commonly used nonprescription analgesic and antipyretic in many developed countries ${ }^{4}$, accidental or intentional overdose with APAP results in lethal liver injury ${ }^{5}$. It is well established that APAP induces hepatic inflammation and liver cell damage 6 . Whether hyperglycemia aggravates APAP-induced acute liver injury remains largely unknown.

Kupffer cells (KCs) are reemerging as critical mediators of both liver injury and repair. KCs exhibit a tremendous plasticity, depending on the local metabolic and immune environment. They can express a range of polarized phenotypes, from the proinflammatory M1 phenotype to the anti-inflammatory M2 phenotype ${ }^{7}$. M1 macrophages are characterized by induction of STAT1 and NF-kB transcription factors, as well as production of proinflammatory cytokines including TNF- $\alpha$, IL-1, IL-6, and IL-12 ${ }^{8}$. M2 macrophages are characterized by activation of transcription factor STAT6, elevated expression of mannose receptor (CD206), and production of cytokines such as TGF- $\beta$, CCL18, and IL-1Ra .

Oxidative stress is caused by intracellular presence of reactive oxygen species (ROS) that overcomes the natural anti-oxidant defense of the cell ${ }^{10}$. Studies had shown that oxidative stress was a major mediator that underlied diabetic complications $^{11,12}$. Diabetic conditions trigger ROS formation in macrophages ${ }^{13}$. The diabetes-induced secretion of monocyte chemotactic protein CCL2 (MCP-1) by endothelial cells attracted monocytes, whereas upregulated endothelial surface expression of adhesion molecule VCAM promoted their adhesion and diapedesis ${ }^{13}$. Transmigrated monocytes differentiated into macrophages and further exacerbated inflammation by mediating tissue injury and secreting proinflammatory cytokines and proteases as well as producing increased ROS levels in the tissues ${ }^{13}$. Studies showed hyperglycemia were closely associated with the production of $\operatorname{ROS}^{14,15}$. Hyperglycemia could mediate retinopathy and kidney damage by inducing ROS production $^{14,15}$. Hyperglycemia caused mitochondrial dysfunction in endothelial cells and macrophages and aberrant activation of cytoplasmic NADPH oxidases (NOX) that together exacerbated ROS production ${ }^{16,17}$. Whereas the mechanism that hyperglycemia increases the production of ROS in KCs against APAP-induced acute liver injury remains unclear.

In this study, we determined that hyperglycemia aggravated acute liver injury by promoting liver-resident macrophage proinflammatory response via AMPK/PI3K/ AKT-induced oxidative stress.

\section{Results}

Hyperglycemia exacerbates APAP-induced acute liver injury

To better study the role of diabetes/hyperglycemia in the development of APAP-induced acute liver injury, we induced type I diabetes in C57/B6J mice with multiple injections of low-dose STZ. Hyperglycemia was confirmed as shown in Fig. 1a. Indeed, compared with CON groups, mice in the STZ groups developed significantly more severe liver injury at $24 \mathrm{~h}$ post treated with APAP, as demonstrated by higher serum alanine aminotransferase (ALT) and aspartate transaminase (AST) levels (Fig. 1b, c), severely damaged liver architecture (Fig. 1d), extensive hepatocellular apoptosis (Fig. 1e, f), and significantly lower levels of antiapoptotic proteins Bcl-2 and Bcl-xL (Fig. 1g). Thus, our results indicated that hyperglycemia increased APAP-induced acute liver injury.

\section{Hyperglycemia enhances KC-related inflammation posttreated with APAP}

Because acute liver injury is intimately related to inflammatory response, $\mathrm{KC}$ relative cytokines and chemokines gene induction were detected in different groups by qRT-PCR. Livers from hyperglycemic mice treated with APAP showed significantly higher levels of proinflammatory TNF- $\alpha$ and IL- 6 , but lower levels of antiinflammatory IL-10 gene induction compared to APAP treatment alone (Fig. 2a). These results were further confirmed by similar serum TNF- $\alpha$, IL-6, and IL-10 levels (Fig. 2b). We furtherly evaluated the effect of hyperglycemia on regulating peripheral macrophage and neutrophil infiltration by immunohistochemical staining. Interestingly, hyperglycemia significantly increased the number of $\mathrm{F} 4 / 80$ + total intrahepatic macrophages, $\mathrm{CD} 11 \mathrm{~b}+$ infiltrating macrophages, and Ly6G + neutrophils in APAP-challenged livers (Fig. 2c-h).

\section{Hyperglycemia mediates KC M1/M2 polarization in response to APAP treatment}

Macrophages can be broadly classified into M1 (proinflammatory) and M2 (anti-inflammatory) subtypes based on function ${ }^{18}$. Thus, we explored the role of hyperglycemia in affecting $\mathrm{KC} \mathrm{M} 1 / \mathrm{M} 2$ polarization. $\mathrm{KCs}$ isolated from hyperglycemic mice posttreated with APAP exhibited higher levels of MCP-1 and inducible NO synthase (iNOS) but much lower levels of Arg1 and CD206 gene induction compared to APAP treatment alone (Fig. 3a). We next analyzed the levels of TNF- $\alpha$, IL-6, and IL-10 in KCs culture supernatant by ELISA. As shown in Fig. 3b, KCs isolated from hyperglycemic mice posttreated with APAP secreted higher levels of the proinflammatory cytokines TNF- $\alpha$ and IL- 6 , and lower levels of the antiinflammatory cytokine IL-10. Hyperglycemia significantly increased the number of KCs positive for iNOS (M1 marker) post APAP treatment compared to APAP alone, but decreased the number of KCs positive for CD206 (M2 marker) (Fig. 3c-f). Furthermore, as shown by western blot in Fig. 3g, hyperglycemic KCs were marked by increased activation of STAT1 but decreased STAT6 


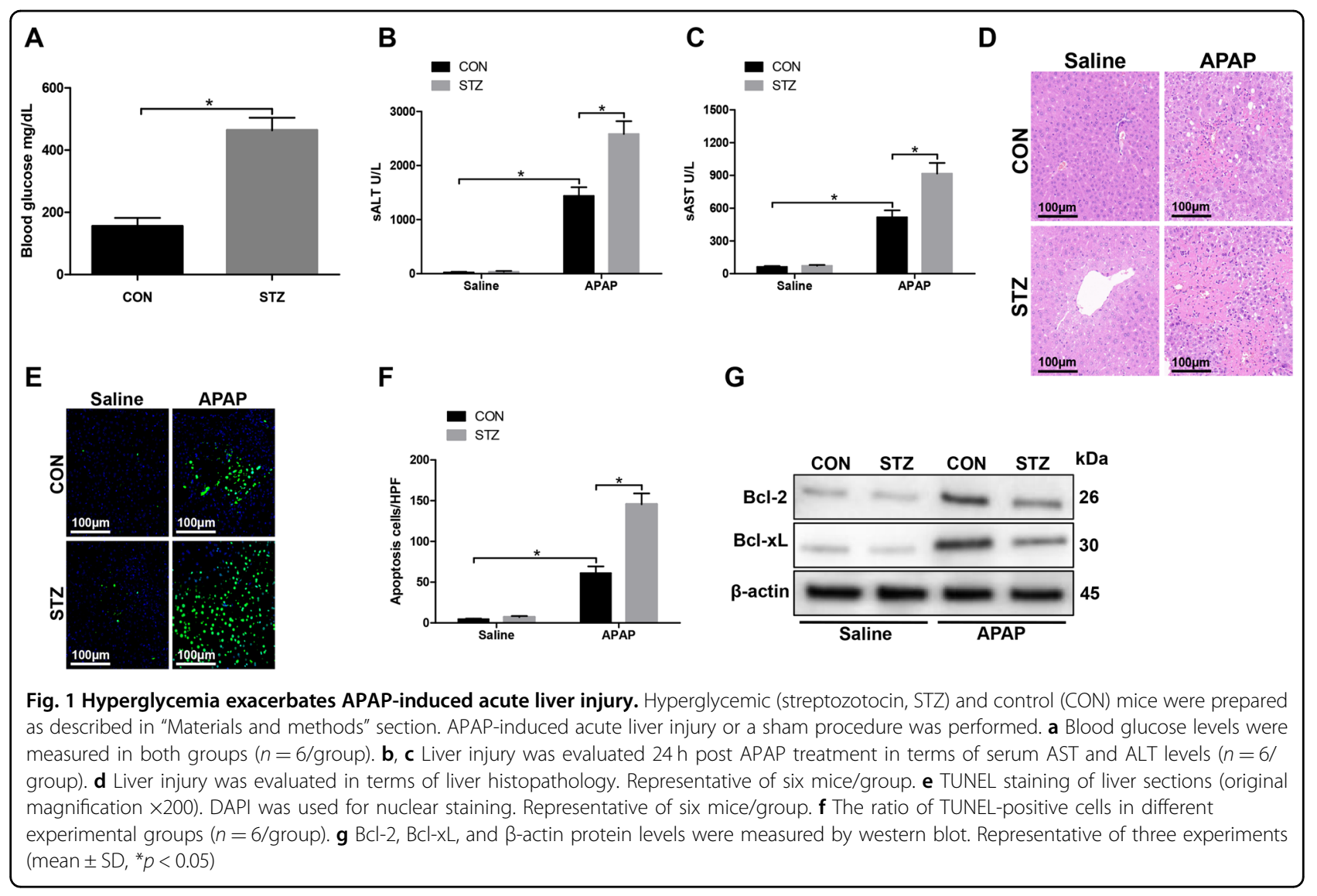

activation post APAP treatment. These results showed that hyperglycemia augmented KC M1 polarization and inhibited KC M2 polarization post APAP treatment.

\section{Hyperglycemia mediates KC M1/M2 polarization by} inducing ROS production against APAP-induced acute liver injury

Many evidences illustrated that hyperglycemia was related to ROS generation ${ }^{14,19}$. Based on these findings, we detected the levels of ROS in KCs from hyperglycemic mice post APAP treatment. The result showed that hyperglycemia significantly increased KCs ROS production (Fig. 4a, b). ROS inhibitor NAC was used to further study the functional role of ROS production in mediating hyperglycemic $\mathrm{KC}$ polarization. As shown in Fig. 4c-e, NAC significantly inhibited ROS production, decreased proinflammatory cytokines TNF- $\alpha$ and IL- 6 and increased anti-inflammatory cytokines IL-10 secretion by KCs from hyperglycemic mice post APAP treatment compared to KCs from normoglycemic mice posttreated with APAP. Furthermore, KCs isolated from hyperglycemic mice posttreated with APAP and NAC exhibited lower levels of MCP-1 and iNOS but much higher levels of Arg1 and CD206 gene induction compared to APAP treatment alone (Fig. 5a). Similarly, NAC significantly decreased the number of KCs positive for iNOS (M1 marker) from hyperglycemic mice post APAP treatment compared to APAP alone, but increased the number of KCs positive for CD206 (M2 marker) (Fig. 5b-e). Furthermore, as shown by western blot in Fig. 5f, NAC decreased STAT1 activation but increased STAT6 activation in KCs from hyperglycemic mice treated with APAP. These results showed that ROS production promoted KC M1 polarization and inhibited KC M2 polarization in hyperglycemic mice post APAP treatment.

\section{Hyperglycemia mediates KC ROS production by inactivating AMPK and inducing PI3K/AKT signaling pathway activation in response to APAP treatment}

To examine the mechanism of hyperglycemia mediating ROS production, we detected the protein levels of $\mathrm{p}$ AMPK and p-AKT, which have been demonstrated associated with ROS generation, in liver and KCs from experimental groups ${ }^{20,21}$. As shown in Fig. 6a, significantly lower protein levels of $\mathrm{p}$-AMPK and higher protein levels of $\mathrm{p}-\mathrm{AKT}$ were found in liver tissues from hyperglycemic mice posttreated with APAP. Similarly, decreased protein levels of p-AMPK and increased protein levels of $\mathrm{p}$-AKT were also found in $\mathrm{KCs}$ from hyperglycemic mice posttreated with APAP (Fig. 6b). To 


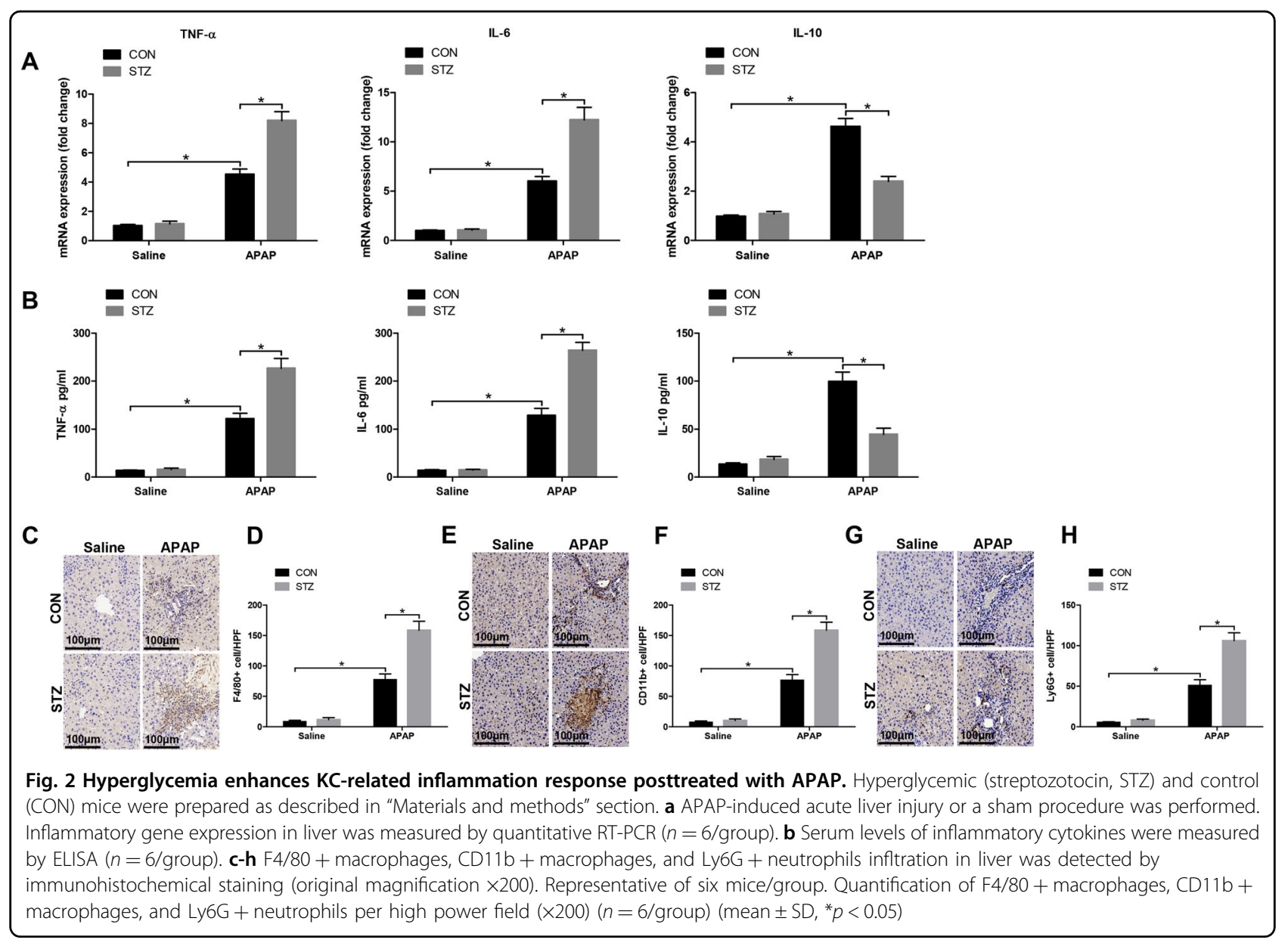

further determine the role of PI3K in regulating hyperglycemic KCs ROS production, we employed PI3K antagonist LY294002 in vitro. Indeed, LY294002 effectively inhibited AKT activation (Fig. 6c). Furthermore, LY294002 significantly inhibited hyperglycemic KCs ROS production posttreated with APAP (Fig. 6d, e). Afterward, AMPK activator AICAR was utilized to determine the relationship between the AMPK and PI3K/AKT signaling pathway. Indeed, AICAR significantly increased protein levels of p-AMPK and decreased protein levels of p-AKT in $\mathrm{KCs}$ isolated from hyperglycemic mice post APAP treatment (Fig. 6f). Moreover, AICAR notably inhibited hyperglycemic KCs ROS production posttreated with APAP (Fig. 6g, h). Interestingly, in vivo AMPK activation alleviated the traumatic role of hyperglycemia in APAPinduced liver injury, as evidenced by lower serum ALT and AST levels (Fig. 7a, b), decreased liver architecture damage (Fig. 7c), and decreased hepatocellular apoptosis (Fig. $7 \mathrm{~d}-\mathrm{f}$ ) compared to APAP treatment alone. Finally, we detected $\mathrm{KC}$ relative cytokines gene induction in different groups by qRT-PCR. Hyperglycemic livers from APAP mice treated with AICAR showed significantly lower levels of proinflammatory TNF- $\alpha$ and IL-6, but higher levels of anti-inflammatory IL-10 gene induction compared to APAP treatment alone (Fig. 8a). These results were further confirmed by similar serum TNF- $\alpha$, IL-6, and IL-10 levels (Fig. 8b). We furtherly evaluated the effect of AICAR on regulating peripheral macrophage and neutrophil infiltration by immunohistochemical staining. Interestingly, AICAR significantly decreased the number of $\mathrm{F} 4 / 80$ + total intrahepatic macrophages, CD11b + infiltrating macrophages, and Ly6G + neutrophils in APAP-challenged livers (Fig. 8c-h).

\section{Discussion}

Diabetes mellitus/hyperglycemia is a major public health problem worldwide, which seriously impairs the quality of life of the patients ${ }^{19,22}$. Chronically untreated diabetes causes various macro- and micro-vascular complications, such as atherosclerosis ${ }^{23}$, diabetic nephropathy $^{24}$, diabetic retinopathy ${ }^{25}$, and neural damage ${ }^{19}$. Our study addressed the question whether and how hyperglycemia impacted APAP-induced acute liver injury, focusing on its effects on the proinflammatory activation 


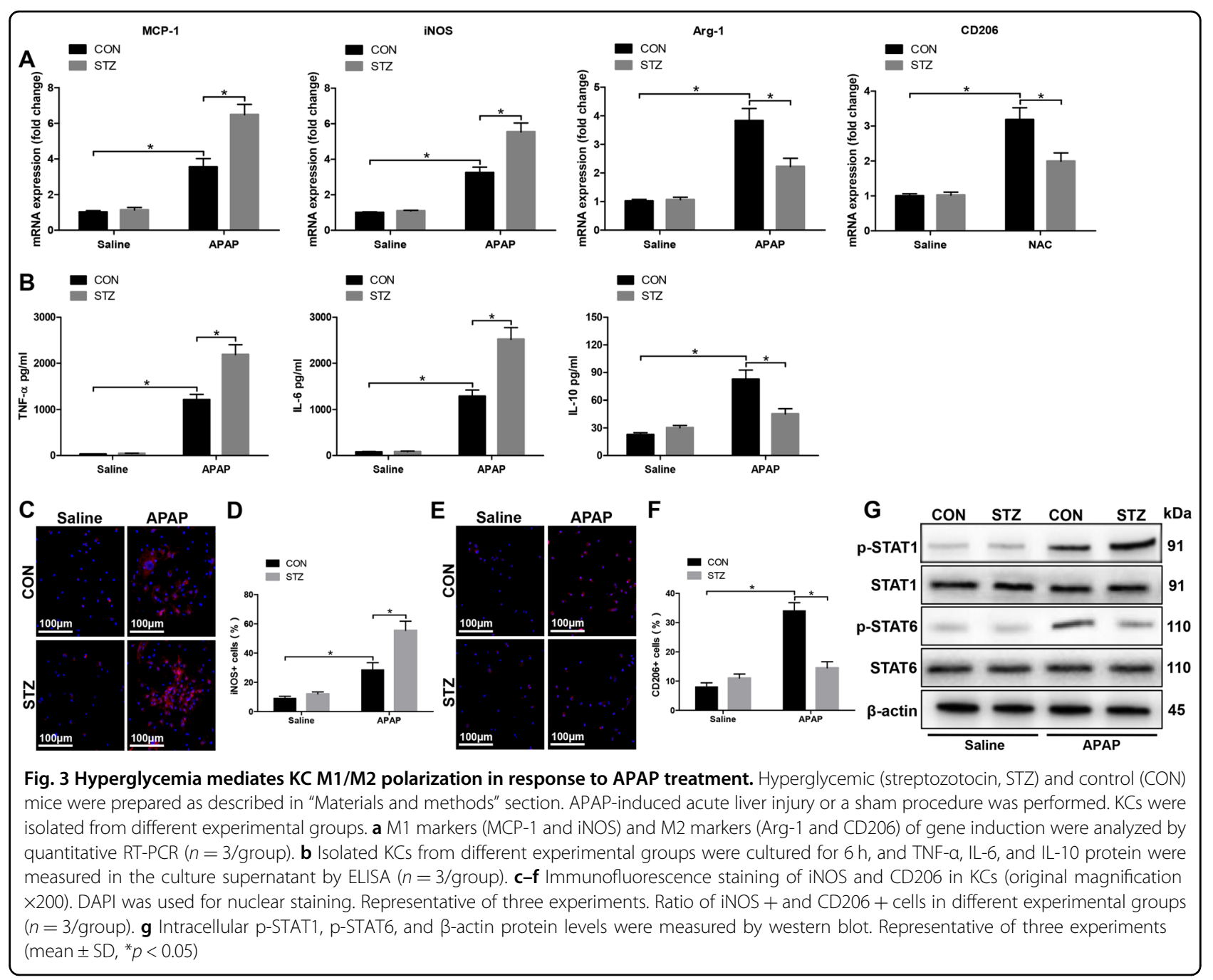

of the liver-resident macrophages (KCs). To the best of our knowledge, this is the first study to demonstrate that hyperglycemia exacerbates APAP-induced acute liver injury by promoting liver-resident macrophage proinflammatory response via AMPK/PI3K/AKT-mediated oxidative stress.

APAP is a commonly used nonprescription analgesic and antipyretic in many developed countries ${ }^{4}$. However, APAP overdose could cause liver injury in animals and humans by inducing mitochondria damage and subsequent necrosis in hepatocytes ${ }^{26}$. Excessive production of ROS was the main pathogenesis of APAP overdose, which was mediated by $\mathrm{N}$-acetyl-p-benzoquinone imine, a metabolite of $\mathrm{APAP}^{27}$. Study demonstrated APAP could trigger an hepatic inflammatory response, including activation of resident macrophages and lymphocytes ${ }^{28-30}$. Other studies showed hyperglycemia aggravated hepatic ischemia and reperfusion injury due to hyperinflammatory immune activation in $\mathrm{KCs}^{2,3}$. These studies showed KCs play a vital role in acute liver injury. However, the effect of hyperglycemia on $\mathrm{KCs}$ against APAP-induced liver inflammation remains unknown.

KCs exhibit a tremendous plasticity, depending on the local metabolic and immune environment. They can express a range of polarized phenotypes, from the proinflammatory M1 phenotype to the anti-inflammatory M2 phenotype 7 . M1 activated macrophages are characterized by increased expression of transcription factors, such as STAT1 and interferon-regulatory factor 5 (IRF$5)^{31}$, and proinflammatory cytokines, including TNF- $\alpha$, IL-6, IL-12, and (iNOS) ${ }^{32}$. While M2 activated macrophages exhibit high expression of transcription factors, including STAT6, IRF-4, and PPAR $\gamma^{31}$, and antiinflammatory mediators, such as IL-10 and IL-1 decoy receptor $^{32}$. Follow-up studies indicated that inactivation of these tissue macrophages with gadolinium chloride and dextran sulfate attenuated the moderate liver injury after APAP treatment ${ }^{33}$. A similar protection together with 


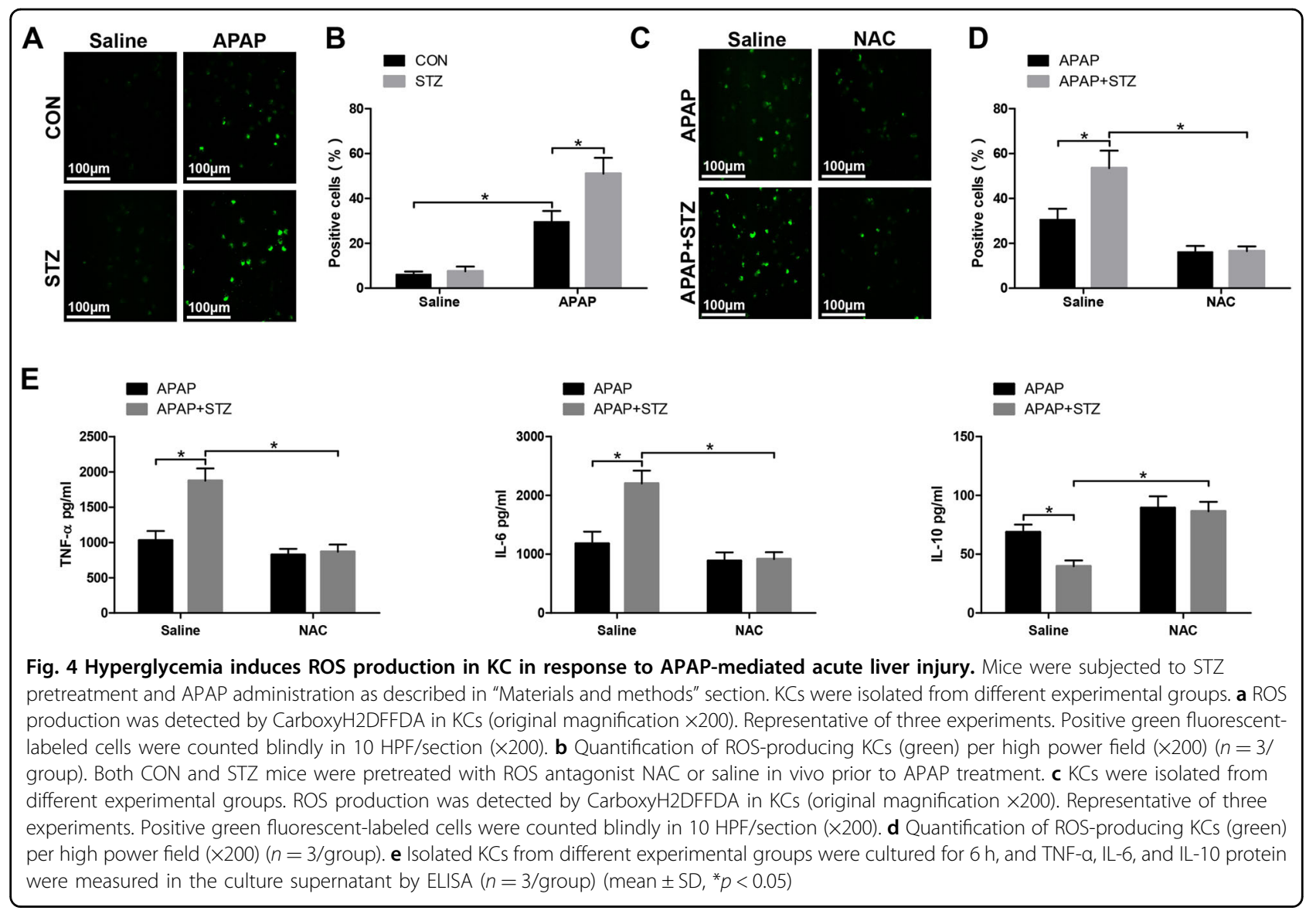

elimination of nitrotyrosine staining was reported after treatment with gadolinium chloride in a mouse model of APAP-induced liver damage ${ }^{34}$. Because gadolinium chloride and related compounds functionally inactivated $\mathrm{KCs}$, that is, mainly impaired their capacity to generate $\operatorname{rROS}^{35,36}$. These data indicated that KCs could be the dominant source of ROS formation after APAP overdose $\mathrm{s}^{34,37}$.

ROS play a pivotal role in the development, growth, and differentiation of multicellular organisms. They are kept in cells at a baseline level that supports cellular proliferation and metabolism, but are also used as key signal transduction molecules that regulate many important metabolic and regulatory pathways in cells ${ }^{38}$. Recent studies showed that oxidative stress was a major mediator that underlied diabetic complications ${ }^{12,39,40}$. Studies showed hyperglycemia triggered ROS formation in macrophages $^{13,19}$. Monocytes were attracted by MCP-1 secreted by endothelium under diabetes ${ }^{13}$. Transmigrated monocytes differentiated into macrophages and produced increased ROS levels, aggravating inflammation and tissues injury ${ }^{13}$. Other studies also showed that hyperglycemia increased ROS production by causing mitochondrial dysfunction in macrophages and aberrant activation of cytoplasmic NADPH oxidases $(\mathrm{NOX})^{16,17}$. In contrast to well-regulated ROS production in antimicrobial response, metabolically generated ROS in diabetic macrophages is more erratic and dysregulated. Its production was associated with promotion of M1-like macrophage phenotype that favors the progression of diabetic complications ${ }^{41}$. It was hypothesized that the metabolism of APAP triggered the oxidant stress ${ }^{42}$. Other studies had confirmed that ROS production induced by mitochondrial oxidative stress played an important role in APAP-induced hepatoxic damage ${ }^{43,44}$.

According to the above studies, we tested how hyperglycemia impacted APAP-induced acute liver injury, focusing on its effects on the proinflammatory activation of KCs. We first detected the levels of ROS in KCs from hyperglycemic mice post APAP treatment. The result showed hyperglycemia increased ROS production and inflammatory response of $\mathrm{KCs}$ against APAP-induced acute liver injury. ROS inhibition by its antagonist NAC effectively suppressed ROS production and inflammatory response of KCs from hyperglycemic mice post APAP treatment. Furthermore, we hypothesized that AMPK could be associated with the PI3K/AKT signaling pathway in $\mathrm{KCs}$ from hyperglycemic mice post APAP treatment 


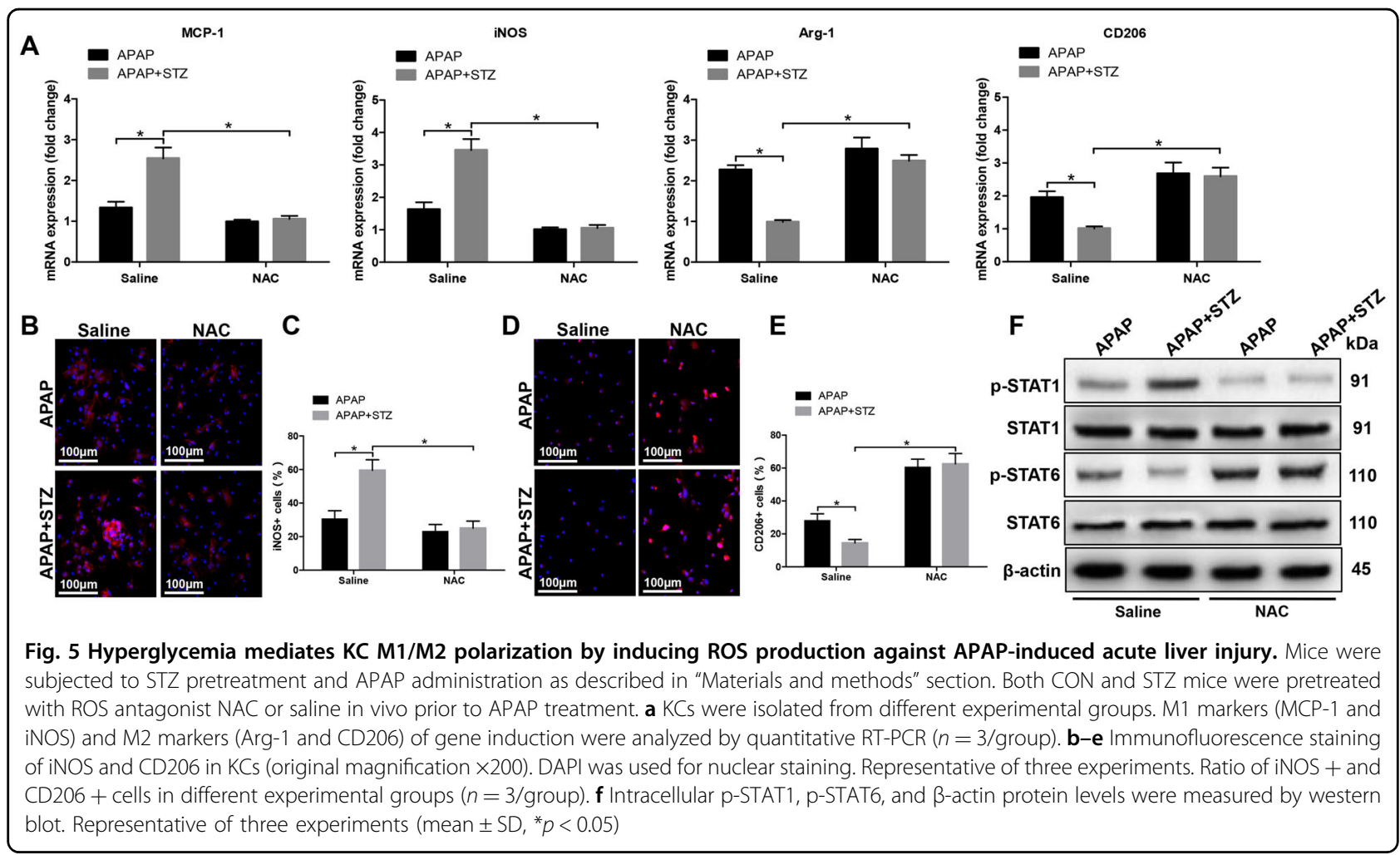

according to the KEGG database. Therefore, we measured protein levels of p-AMPK, AMPK, p-AKT, and AKT. We found hyperglycemia increased $\mathrm{p}$-AMPK protein expression while decreasing $\mathrm{p}$-AKT protein expression. PI3K inhibition by its antagonist LY294002 or AMPK activation by its agonist AICAR inhibited AKT activation, and subsequently, inhibited ROS production in $\mathrm{KCs}$, leading to ultimately reduced APAP-induced liver injury in the hyperglycemic mice. These results showed hyperglycemia increased ROS production by inhibiting AMPK activation and inducing PI3K/AKT signaling pathway activation.

In conclusion, our results demonstrated that hyperglycemia exacerbated APAP-induced acute liver injury by promoting liver-resident macrophage proinflammatory response via AMPK/PI3K/AKT-mediated oxidative stress. These findings indicated that inhibition of oxidative stress could be a novel therapeutic approach to mitigate APAPinduced hepatotoxicity and liver injury in hyperglycemia.

\section{Materials and methods}

\section{Animals}

Male wild-type C57BL/6 J mice (6-8 weeks old) were purchased from the Laboratory of Animal Resources of Nanjing Medical University and were housed under specific pathogen-free conditions with access to properly sterilized water and food. This study was performed in strict accordance with the recommendations in the protocol (number NMU08-092) approved by the
Institutional Animal Care and Use Committee of Nanjing Medical University.

\section{Mouse hyperglycemia and APAP-induced acute liver injury model}

Streptozotocin (STZ) or vehicle control (sodium citrate buffer) was injected intraperitoneally (i.p.) into separate groups of 6 -week-old mice at $40 \mathrm{mg} / \mathrm{kg}$ for five consecutive days. Blood glucose levels from the tail vein were tested at day 14 (9 days after the last shot). Mice with blood glucose over $300 \mathrm{mg} / \mathrm{dL}$ were considered hyperglycemic (STZ group). The vehicle control group (CON group) was subject to the same intraperitoneal injection procedure, but with sodium citrate buffer. The mice were intraperitoneally injected with a dose of $400 \mathrm{mg} / \mathrm{kg}$ APAP (Sigma, St. Louis, MO, USA) dissolved in saline. The control mice received the same volume of saline via intraperitoneal injection. The mice were killed $24 \mathrm{~h}$ after APAP treatment. Serum and liver samples were collected. Tissues were stored continuously in liquid $\mathrm{N}_{2}$. Sections from the dissected livers were also fixed in $10 \%$ neutral buffered formalin for histological analysis.

\section{Hepatocellular function assay}

Blood samples were collected and centrifuged to obtain serum for analysis. Serum levels of ALT and AST were detected by an automated chemical analyzer (Olympus Company, Tokyo, Japan). 


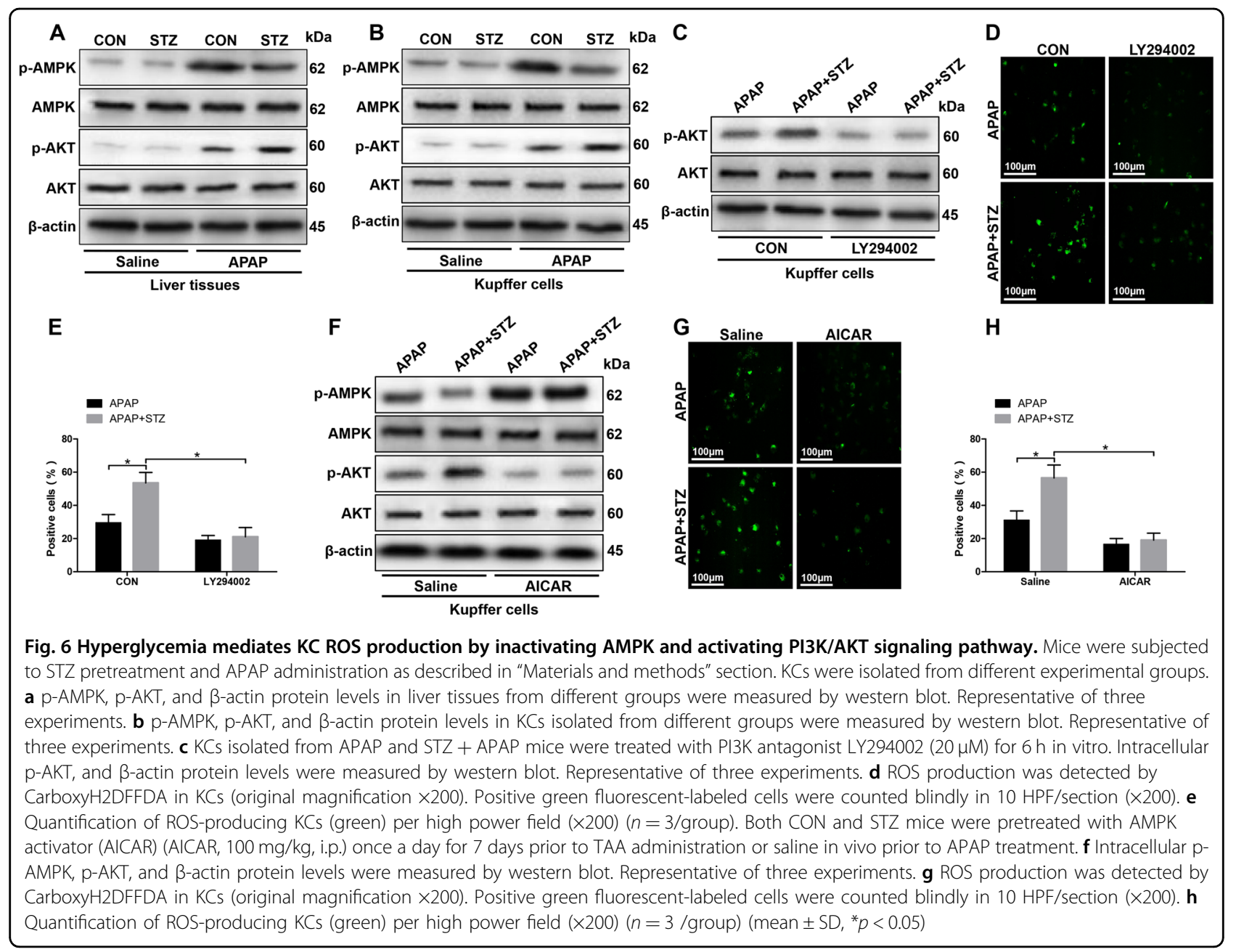

\section{TUNEL staining}

TUNEL staining of liver tissues was performed using a fluorescent detection kit (Roche, Switzerland) according to the manufacturer's instructions. Briefly, paraffin sections of hepatic tissues ( $4 \mu \mathrm{m}$ thickness) were deparaffinated in toluene and then dehydrated by a graded series of ethanol solutions (ethanol 100, 95, 90, 80, and $70 \%)$ and washed in distilled water. They were then incubated with $20 \mu \mathrm{g} / \mathrm{ml}$ proteinase $\mathrm{K}$ solution (Roche, Switzerland) for $25 \mathrm{~min}$ at $37^{\circ} \mathrm{C}$. The tissues were then washed with PBS three times for $3 \mathrm{~min}$. After washing with PBS, the tissues were then incubated with permeabilize working solution at $37^{\circ} \mathrm{C}$ for $20 \mathrm{~min}$. The tissues were then washed with PBS three times for $3 \mathrm{~min}$. Next, the tissues were incubated with TUNEL solution (TdT: $\mathrm{dUTP}=1: 9)$ at $37^{\circ} \mathrm{C}$ for $2 \mathrm{~h}$ in a moist and dark environment. The tissues were then washed with PBS three times for $3 \mathrm{~min}$ and incubated with DAPI solution at $37^{\circ} \mathrm{C}$ for $10 \mathrm{~min}$ in a dark environment. Then the tissues were washed with PBS three times for $3 \mathrm{~min}$. Sections were photographed by Fluorescent Microscopy. DAPI glows blue by UV excitation wavelength $330-380 \mathrm{~nm}$ and emission wavelength $420 \mathrm{~nm}$; FITC glows green by excitation wavelength $465-495 \mathrm{~nm}$ and emission wavelength $515-555 \mathrm{~nm}$. The nucleus is blue by labeling with DAPI. Tunel assay kit is from Roch and labeled with FITC. Positive apoptosis cells are green.

\section{Histopathology, immunohistochemical staining, and immunofluorescence staining}

Liver tissues were collected and stained with hematoxylin and eosin, and light microscopy was used to observe inflammation and tissue damage. Liver macrophages and neutrophils were detected using primary rat anti-mouse F4/80, CD11b, and Ly6G mAb, respectively (BD Biosciences, San Jose, CA, USA). The secondary, biotinylated goat anti-rat IgG (Vector, Burlingame, CA, USA) was incubated with immunoperoxidase (ABC Kit, Vector), according to the manufacturer's instruction. Positive cells were counted blindly in $10 \mathrm{HPF} /$ section. iNOS and CD206 in KCs were identified by immunofluorescence using rabbit anti-mouse iNOS $\mathrm{mAb}$ and anti-mouse 

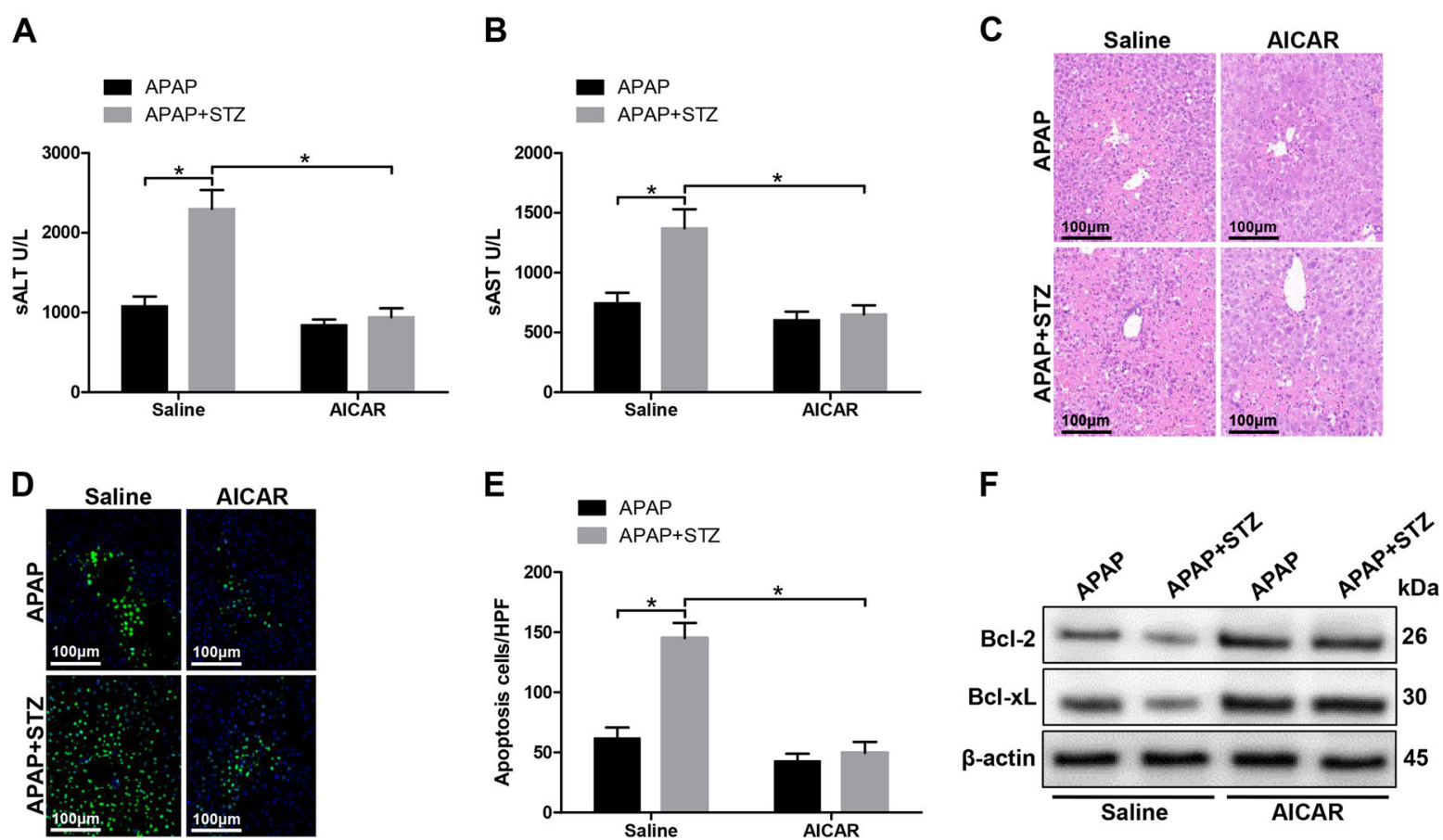

Fig. 7 AMPK activator (AICAR) attenuates APAP-induced acute liver injury under hyperglycemia. Mice were subjected to STZ pretreatment and APAP administration as described in "Materials and methods" section. Both CON and STZ mice were pretreated with AMPK activator (AICAR) or saline in vivo prior to APAP treatment. $\mathbf{a}$, $\mathbf{b}$ Liver injury was evaluated $24 \mathrm{~h}$ post APAP treatment in terms of serum AST and ALT levels ( $n=6 / \mathrm{group}$ ). $\mathbf{c}$ Liver injury was evaluated in terms of liver histopathology. Representative of six mice/group. $\mathbf{d}$ TUNEL staining of liver sections (original magnification $\times 200$ ). DAPI was used for nuclear staining. Representative of six mice/group. e The ratio of TUNEL-positive cells in different experimental groups ( $n=$ 6/group). $\mathbf{f} \mathrm{BCl}-2, \mathrm{BCl}-\mathrm{xL}$, and $\beta$-actin protein levels were measured by western blot. Representative of three experiments (mean $\left.\pm S D,{ }^{*} p<0.05\right)$

CD206 mAb (Cell Signaling Technology, MA, USA). After incubation with secondary goat anti-mouse Texas Red-conjugated IgG (Sigma, St.Louis, MO, USA), KCs were premounted with VECTASHIELD medium with DAPI (Vector). Negative control slided with the primary antibodies omitted were included in all assays. Positive cells were blindly observed in $10 \mathrm{HPF} /$ section $(\times 200)$.

\section{Cell isolation and culture}

KCs were isolated as previously described ${ }^{45}$. Briefly, livers were perfused in situ via the portal vein with calcium- and magnesium-free HBSS supplemented with $2 \%$ heat-inactivated FBS, followed by $0.27 \%$ collagenase IV (Sigma, St. Louis, MO). Perfused livers were dissected and teased through $70 \mathrm{~mm}$ nylon mesh cell strainers (BD Biosciences, San Diego, CA), followed by suspension in $40 \mathrm{ml}$ of DMEM supplemented with $10 \%$ FBS, $10 \mathrm{mM}$ HEPES, $2 \mathrm{mM}$ GlutaMax, $100 \mathrm{U} / \mathrm{ml}$ penicillin, and $100 \mathrm{mg} / \mathrm{ml}$ streptomycin for $15 \mathrm{~min}$ at $37^{\circ} \mathrm{C}$. Nonadherent cells were removed by replacing the culture medium. The adherent cells were used for further ex vivo experiments. KCs were cultured in vitro for $6 \mathrm{~h}$, and then cells or supernatants were collected for further analysis. For M2 polarization, KCs isolated from mice were treated with $10 \mathrm{ng} / \mathrm{ml} \mathrm{IL-4} \mathrm{(Sigma,} \mathrm{Saint} \mathrm{Louis,}$ MO, USA).

\section{Western blot analysis}

Liver tissues and cellular proteins were extracted with ice-cold lysis buffer $[1 \%$ Triton X-100, $0.5 \%$ sodium deoxycholate, $0.1 \%$ SDS, $10 \%$ glycerol, $137 \mathrm{mM}$ sodium chloride, and $20 \mathrm{mM}$ Tris ( $\mathrm{pH}$ 7.4)]. Proteins $(20 \mu \mathrm{g})$ were separated by $10 \%$ SDS-PAGE and transferred to polyvinylidene difluoride nitrocellulose membranes. PhosphoSTAT1, STAT1, phospho-STAT6, STAT6, phosphoAMPK, AMPK, phospho-AKT, AKT, Bcl-2, Bcl-xl, and $\beta$-actin rabbit mAbs (Cell Signaling Technology, MA, USA) were used. HRP-conjugated goat anti-rabbit IgG (Cell Signaling Technology, MA, USA) was used as the secondary antibody.

\section{Quantitative RT-PCR analysis}

Total RNA was extracted from frozen liver tissues and cells using TRIZOL reagent (Invitrogen, Carlsbad, CA, USA) and was reverse-transcribed into cDNA using the Transcriptor First Strand cDNA Synthesis Kit (Roche, 


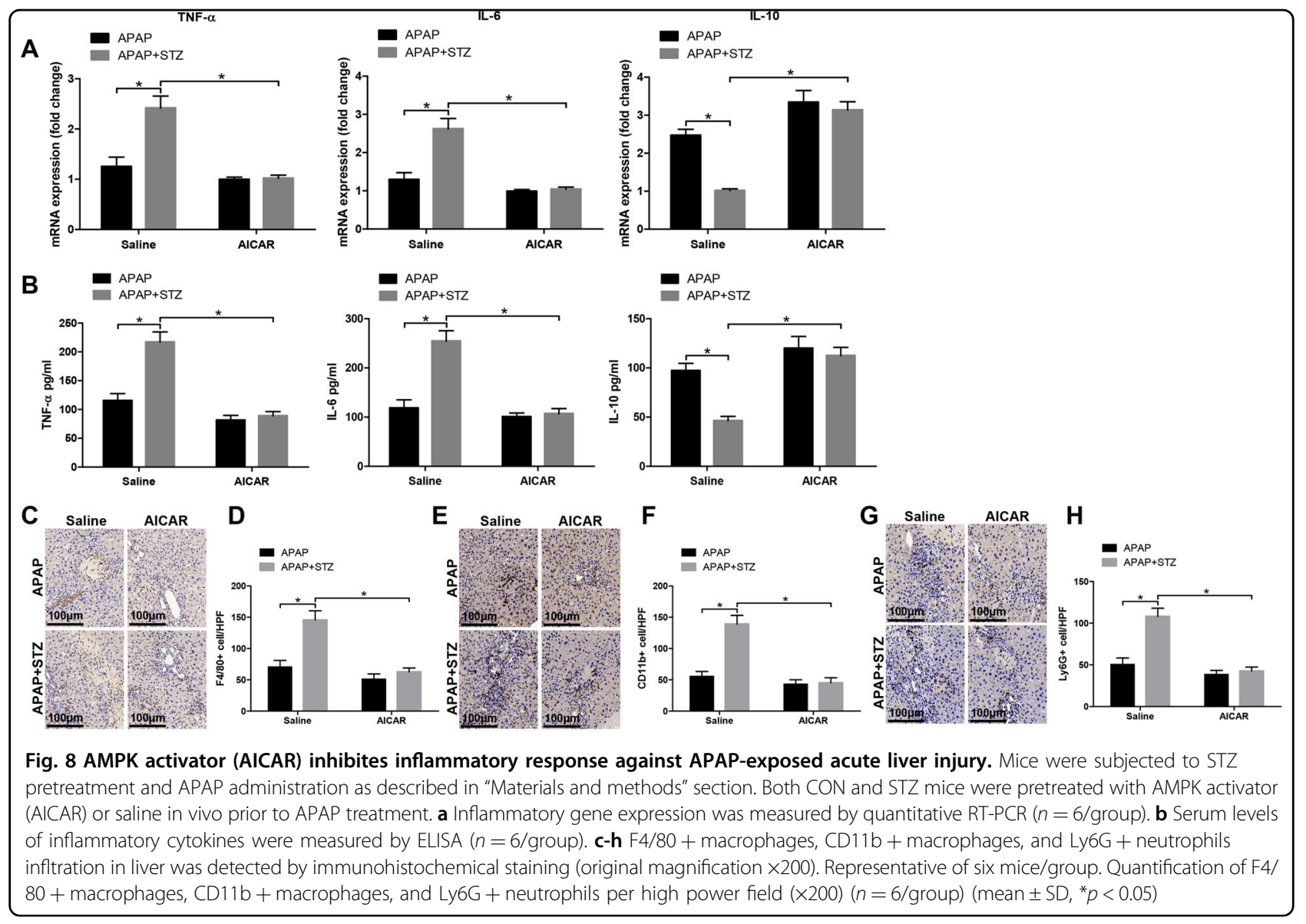

Table 1 Primer sequences for the amplification

\begin{tabular}{|c|c|c|}
\hline Gene & Forward primer $\left(5^{\prime} \rightarrow 3^{\prime}\right)$ & Reverse primer $\left(5^{\prime} \rightarrow 3^{\prime}\right)$ \\
\hline TNF-a & 5'-GACGTGGAACTGGCAGAAGAG-3' & 5'-TTGGTGGTTTGTGAGTGTGAG-3' \\
\hline IL-6 & 5'-CCAAGAGGTGAGTGCTTCCC-3' & 5'-CTGTTGTTCAGACTCTCTCCCT-3' \\
\hline |L-10 & 5'-GCTCTTACTGACTGGCATGAG-3' & 5'-CGCAGCTCTAGGAGCATGTG-3' \\
\hline MCP-1 & 5'-TTAAAAACCTGGATCGGAACCAA-3' & 5'-GCATTAGCTTCAGATTTACGGGT-3' \\
\hline iNOS & 5'-GTTCTCAGCCCAACAATACAAGA-3' & 5'-GTGGACGGGTCGATGTCAC-3' \\
\hline Arg-1 & 5'-CTCCAAGCCAAAGTCCTTAGAG-3' & 5'-AGGAGCTGTCATTAGGGACATC-3' \\
\hline CD206 & 5'-CTCTGTTCAGCTATTGGACGC-3' & 5'-CGGAATTTCTGGGATTCAGCTTC-3' \\
\hline HPRT & 5'-TCAGTCAACGGGGGACATAAA-3' & 5'-GGGGCTGTACTGCTTAACCAG-3' \\
\hline
\end{tabular}

Indianapolis, IN, USA). Quantitative real-time PCR was performed using SYBR green (Roche, Indianapolis, IN, USA) on a StepOnePlus Real-Time PCR System (Applied Biosystems, Foster City, CA). PCR cycling conditions were as follows: $95^{\circ} \mathrm{C}$ for $30 \mathrm{~s} ; 40$ cycles of $95^{\circ} \mathrm{C}$ for $5 \mathrm{~s}$ and $60{ }^{\circ} \mathrm{C}$ for $30 \mathrm{~s}$; and dissociation at $95^{\circ} \mathrm{C}$ for $15 \mathrm{~s}, 60^{\circ} \mathrm{C}$ for $60 \mathrm{~s}$, and $95^{\circ} \mathrm{C}$ for $15 \mathrm{~s}$. qRT-PCR reactions were all repeated three times. The expression levels of target genes and the results were normalized against HPRT expression.
Primer sequences used for the amplification were shown in Table 1.

\section{ROS assay}

ROS production in the KCs was measured using the Carboxy-H2DFFDA kit (Thermo Fisher Scientific, Waltham, MA). ROS production by the KCs was analyzed and quantified by confocal microscopy (ZEISS, Oberkochen, Germany) according to the manufacturer's instructions. 


\section{ELISA}

TNF- $\alpha$, IL-6, and IL-10 levels in serum or cell culture supernatants were measured using an ELISA kit (eBiosciences, San Diego, CA, USA) according to the manufacturer's protocols.

\section{Statistical analysis}

Data were expressed as the mean $\pm S D$ and analyzed using the Permutation $t$-test and Pearson correlation. Multiple group comparisons were performed using oneway ANOVA followed by Bonferroni's post hoc test. All analysis were performed using Stata software (version 11.0). $P<0.05$ (two-tailed) was considered statistically significant.

\section{Acknowledgements}

This study was supported by grants from the National Natural Science Foundation of China 81571564, 81100270, 91442117, 1310108001, and 81210108017, the National Science Foundation of Jiangsu Province BK20131024 and BE2016766, the 863 Young Scientists Special Fund grant SS2015AA0209322, and the Foundation of Jiangsu Collaborative Innovation Center of Biomedical Functional Materials.

\section{Author contributions}

Q.W., S.W., H.Z., G.S., X.Gan., Z.S., J.Q., and C.S. performed the experiments. Q.W. and S.W. analyzed the data. Q.W. and S.W. wrote the first draft of manuscript. H. Z., G.S., X.Gan., Z.S., J.Q., and C.S. participated in data analysis and critical discussion. L.L. contributed to the study concept, research design, and finalized the manuscript. All authors were involved in data interpretation, technical support, and writing the paper, and had final approval of the submitted and published versions.

\section{Conflict of interest}

The authors declare that they have no conflict of interest.

\section{Publisher's note}

Springer Nature remains neutral with regard to jurisdictional claims in published maps and institutional affiliations.

Received: 2 May 2019 Revised: 4 June 2019 Accepted: 11 June 2019 Published online: 19 July 2019

\section{References}

1. Mendes-Braz, M. \& Martins, J. O. Diabetes mellitus and liver surgery: the effect of diabetes on oxidative stress and inflammation. Mediat. Inflamm. 2018, 2456579 (2018).

2. Rao, Z. et al. Hyperglycemia aggravates hepatic ischemia and reperfusion injury by inhibiting liver-resident macrophage M2 polarization via C/EBP homologous protein-mediated endoplasmic reticulum stress. Front Immunol. 8, 1299 (2017).

3. Yue, S. et al. Hyperglycemia and liver ischemia reperfusion injury: a role for the advanced glycation endproduct and its receptor pathway. Am. J. Transplant. 15, 2877-2887 (2015).

4. Tsutsui, H. \& Nishiguchi, S. Importance of kupffer cells in the development of acute liver injuries in mice. Int. J. Mol. Sci. 15, 7711-7730 (2014).

5. Larson, A. M. et al. Acetaminophen-induced acute liver failure: results of a United States multicenter, prospective study. Hepatology 42, 1364-1372 (2005).

6. Imaeda, A. B. et al. Acetaminophen-induced hepatotoxicity in mice is dependent on Tlr9 and the Nalp3 inflammasome. J. Clin. Invest. 119, 305-314 (2009).
7. Dixon, L. J., Barnes, M., Tang, H., Pritchard, M. T. \& Nagy, L. E. Kupffer cells in the liver. Compr. Physiol. 3, 785-797 (2013).

8. Van Ginderachter, J. A. et al. Classical and alternative activation of mononuclear phagocytes: picking the best of both worlds for tumor promotion. Immunobiology 211, 487-501 (2006).

9. Gordon, S. \& Martinez, F. O. Alternative activation of macrophages: mechanism and functions. Immunity 32, 593-604 (2010).

10. Schieber, M. \& Chandel, N. S. ROS function in redox signaling and oxidative stress. Curr. Biol. 24, R453-R462 (2014).

11. Pitocco, D., Tesauro, M., Alessandro, R., Ghirlanda, G. \& Cardillo, C. Oxidative stress in diabetes: implications for vascular and other complications. Int J. Mol. Sci. 14, 21525-21550 (2013).

12. Giacco, F. \& Brownlee, M. Oxidative stress and diabetic complications. Circ. Res. 107, 1058-1070 (2010).

13. Tesch, G. H. Role of macrophages in complications of type 2 diabetes. Clin. Exp. Pharm. Physiol. 34, 1016-1019 (2007).

14. Wang, L. et al. Hyperglycemia induces neutrophil extracellular traps formation through an nadph oxidase-dependent pathway in diabetic retinopathy. Front Immunol. 9, 3076 (2018).

15. Chowdhury, S., Ghosh, S., Das, A. K. \& Sil, P. C. Ferulic acid protects hyperglycemia-induced kidney damage by regulating oxidative insult, inflammation and autophagy. Front Pharmacol. 10, 27 (2019).

16. Widlansky, M. E. et al. Altered mitochondrial membrane potential, mass, and morphology in the mononuclear cells of humans with type 2 diabetes. Transl. Res. 156, 15-25 (2010).

17. Huang, X. et al. Augmented NADPH oxidase activity and p22phox expression in monocytes underlie oxidative stress of patients with type 2 diabetes mellitus. Diabetes Res Clin. Pract. 91, 371-380 (2011).

18. Wynn, T. A., Chawla, A. \& Pollard, J. W. Macrophage biology in development, homeostasis and disease. Nature 496, 445-455 (2013).

19. Rendra, E. et al. Reactive oxygen species (ROS) in macrophage activation and function in diabetes. Immunobiology. 224, 242-253 (2019)

20. Rabinovitch, R. C. et al. AMPK maintains cellular metabolic homeostasis through regulation of mitochondrial reactive oxygen species. Cell Rep. 21, 1-9 (2017).

21. Zhao, Y. et al. ROS signaling under metabolic stress: cross-talk between AMPK and AKT pathway. Mol. Cancer 16, 79 (2017).

22. Kim, C. H. Microbiota or short-chain fatty acids: which regulates diabetes? Cell Mol. Immunol. 15, 88-91 (2018).

23. Gray, S. P. et al. NADPH oxidase 1 plays a key role in diabetes mellitusaccelerated atherosclerosis. Circulation 127, 1888-1902 (2013).

24. Bichet, D. G. \& Lussier, Y. Mice deficient for ERAD machinery component Sel1L develop central diabetes insipidus. J. Clin. Invest. 127, 3591-3593 (2017).

25. Retnakaran, R. \& Shah, B. R. Role of type 2 diabetes in determining retinal, renal, and cardiovascular outcomes in women with previous gestational diabetes mellitus. Diabetes Care. 40, 101-108 (2017).

26. Ni, H. M., Bockus, A., Boggess, N., Jaeschke, H. \& Ding, W. X. Activation of autophagy protects against acetaminophen-induced hepatotoxicity. Hepatology 55, 222-232 (2012)

27. James, L. P., Mayeux, P. R. \& Hinson, J. A. Acetaminophen-induced hepatotoxicity. Drug Metab. Dispos. 31, 1499-1506 (2003).

28. Jaeschke, H. Role of inflammation in the mechanism of acetaminopheninduced hepatotoxicity. Expert Opin. Drug Metab. Toxicol. 1, 389-397 (2005).

29. Jaeschke, $\mathrm{H}$. Mechanisms of sterile inflammation in acetaminophen hepatotoxicity. Cell Mol. Immunol. 15, 74-75 (2018).

30. Zhang, C. et al. Macrophage-derived IL-1alpha promotes sterile inflammation in a mouse model of acetaminophen hepatotoxicity. Cell Mol. Immunol. 15, 973-982 (2018).

31. Lawrence, T. \& Natoli, G. Transcriptional regulation of macrophage polarization: enabling diversity with identity. Nat. Rev. Immunol. 11, 750-761 (2011).

32. Gordon, S. \& Taylor, P. R. Monocyte and macrophage heterogeneity. Nat. Rev Immunol. 5, 953-964 (2005).

33. Laskin, D. L., Gardner, C. R., Price, V. F. \& Jollow, D. J. Modulation of macrophage functioning abrogates the acute hepatotoxicity of acetaminophen. Hepatology 21, 1045-1050 (1995)

34. Michael, S. L., Pumford, N. R., Mayeux, P. R., Niesman, M. R. \& Hinson, J. A. Pretreatment of mice with macrophage inactivators decreases acetaminophen hepatotoxicity and the formation of reactive oxygen and nitrogen species. Hepatology 30, 186-195 (1999). 
35. Liu, P. et al. Activation of kupffer cells and neutrophils for reactive oxygen formation is responsible for endotoxin-enhanced liver injury after hepatic ischemia. Shock 3, 56-62 (1995).

36. Jaeschke, H. \& Farhood, A. Neutrophil and kupffer cell-induced oxidant stress and ischemia-reperfusion injury in rat liver. Am. J. Physiol. 260, G355-G362 (1991).

37. Jaeschke, H. et al. Mechanisms of hepatotoxicity. Toxicol. Sci. 65, 166-176 (2002).

38. Zandalinas, S. I. \& Mittler, R. ROS-induced ROS release in plant and animal cells. Free Radic. Biol. Med. 122, 21-27 (2018).

39. Kowluru, R. A., Kowluru, A., Mishra, M. \& Kumar, B. Oxidative stress and epigenetic modifications in the pathogenesis of diabetic retinopathy. Prog. Retin Eye Res. 48, 40-61 (2015).

40. Rajesh, M. et al. Cannabidiol attenuates cardiac dysfunction, oxidative stress, fibrosis, and inflammatory and cell death signaling pathways in diabetic cardiomyopathy. J. Am. Coll. Cardiol. 56, 2115-2125 (2010).
41. Kumar, P., Swain, M. M. \& Pal, A. Hyperglycemia-induced inflammation caused down-regulation of 8-oxoG-DNA glycosylase levels in murine macrophages is mediated by oxidative-nitrosative stress-dependent pathways. Int J. Biochem Cell Biol. 73, 82-98 (2016).

42. Wendel, A., Feuerstein, S. \& Konz, K. H. Acute paracetamol intoxication of starved mice leads to lipid peroxidation in vivo. Biochem. Pharmacol. 28 2051-2055 (1979).

43. Gao, Y. et al. Early stage functions of mitochondrial autophagy and oxidative stress in acetaminophen-induced liver injury. J. Cell Biochem. 118, 3130-3141 (2017).

44. Han, J. et al. Autophagy induced by AXL receptor tyrosine kinase alleviates acute liver injury via inhibition of NLRP3 inflammasome activation in mice. Autophagy 12, 2326-2343 (2016).

45. Zhou, S. et al. Spermine alleviates acute liver injury by inhibiting liver-resident macrophage pro-inflammatory response through ATG5-dependent autophagy. Front Immunol. 9, 948 (2018). 